\title{
Integrating Lie algebroids via stacks
}

\author{
Hsian-Hua Tseng and Chenchang Zhu
}

\begin{abstract}
Lie algebroids cannot always be integrated into Lie groupoids. We introduce a new structure, 'Weinstein groupoid', which may be viewed as stacky groupoids. We use this structure to present a solution to the integration problem of Lie algebroids. It turns out that every Weinstein groupoid has a Lie algebroid and every Lie algebroid can be integrated into such a groupoid.
\end{abstract}

\section{Introduction}

In this paper, we present a new viewpoint to integrate (finite-dimensional) Lie algebroids: unlike (finite-dimensional) Lie algebras which always have their associated Lie groups, Lie algebroids do not always have their associated Lie groupoids [AM84, AM85]. So the Lie algebroid version of Lie's third theorem poses the question indicated by the following chart.

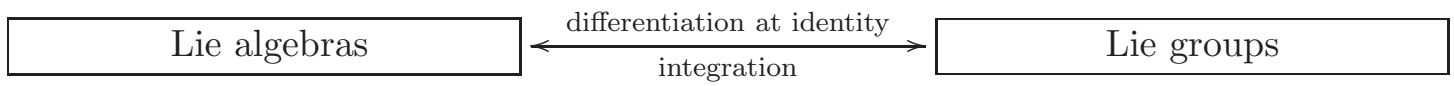

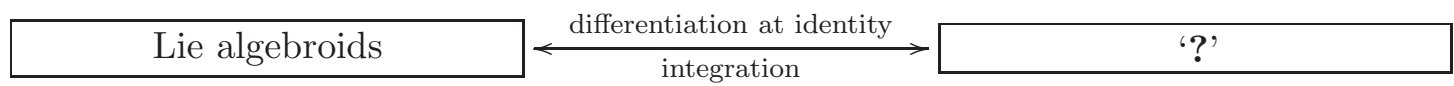

Pradines posed the above question in [Pra68] and constructed local Lie groupoids (formulated in [CDW87, Kar86, van84]) as the integration object '?'. However, a global object for '?' is still required: not only would it give a conceptually better answer to the diagram above (Lie groups are global objects), but it also has profound applications in Poisson geometry, such as Weinstein's symplectic groupoids [Wei87], Xu's Morita equivalence of Poisson manifolds [Xu91b, Xu91a], symplectic realizations [Wei83], Picard groups [BW04] and the linearization problem of Poisson manifolds [CF04].

After Pradines' local groupoids, progress towards special cases of the above integration problem was made by [Daz90, Deb00, Mac87, Nis00, Wei89], among others. An important approach to finding a global object is the use of path spaces. This idea is not new, see [Wei03] for a nice discussion. We pay particular attention to the recent work of Crainic and Fernandes [CF03] and of Cattaneo and Felder [CF01]. For a Lie algebroid $A$, they study the space of $A$-paths. They are able to give a negative answer to the integrability problem: not every Lie algebroid can be integrated into a Lie groupoid. From the space of $A$-paths they construct a topological groupoid and determine equivalent conditions for this groupoid to be a Lie groupoid that integrates the given Lie algebroid $A$. So their work shows that every Lie algebroid can be integrated into a topological groupoid, but in general this topological groupoid does not have enough information to recover the Lie algebroid we start with. As conjectured by Weinstein, one hopes that there are additional structures on this topological groupoid, which allow us to recover the Lie algebroid. As the topological groupoid is a space of leaves, one natural approach is via étale groupoids [Hae84]. It turns out that differentiable stacks discussed

Received 18 May 2004, accepted in final form 25 May 2005.

2000 Mathematics Subject Classification 58H05 (primary), 14A20 (secondary).

Keywords: Lie theory, Lie algebroids, stacks, groupoids.

This journal is (c) Foundation Compositio Mathematica 2006. 


\section{H.-H. Tseng And C. Zhu}

in recent papers [BX, Met03, Pro96] also provide a suitable structure to the above conjecture posed by Weinstein.

We introduce the notion of Weinstein groupoid, which formalizes the additional structures to put on this topological groupoid. By allowing Weinstein groupoids, we answer the integrability problem positively - every Lie algebroid can be integrated into a Weinstein groupoid.

Definition 1.1 (Weinstein groupoid). A Weinstein groupoid over a manifold $M$ consists of the following data:

(i) an étale differentiable stack $\mathcal{G}$ (see $\S 3$ for the definition);

(ii) (source and target) maps $\overline{\mathbf{s}}, \overline{\mathbf{t}}: \mathcal{G} \rightarrow M$, which are surjective submersions between differentiable stacks;

(iii) (multiplication) a map $\bar{m}: \mathcal{G} \times_{\overline{\mathbf{s}}, \overline{\mathbf{t}}} \mathcal{G} \rightarrow \mathcal{G}$, satisfying the following properties:

- $\overline{\mathbf{t}} \circ \bar{m}=\overline{\mathbf{t}} \circ p r_{1}, \overline{\mathbf{s}} \circ \bar{m}=\overline{\mathbf{s}} \circ p r_{2}$, where $p r_{i}: \mathcal{G} \times_{\overline{\mathbf{s}}, \overline{\mathbf{t}}} \mathcal{G} \rightarrow \mathcal{G}$ is the $i$ th projection $\mathcal{G} \times_{\overline{\mathbf{s}}, \overline{\mathbf{t}}} \mathcal{G} \rightarrow \mathcal{G}$;

- associativity up to a 2-morphism, i.e. there is a unique 2-morphism $\alpha$ between maps $\bar{m} \circ$ $(\bar{m} \times i d)$ and $\bar{m} \circ(i d \times \bar{m})$;

(iv) (identity section) an injective immersion $\bar{e}: M \rightarrow \mathcal{G}$ such that, up to 2-morphisms, the following identities

$$
\bar{m} \circ((\bar{e} \circ \overline{\mathbf{t}}) \times i d)=i d, \bar{m} \circ(i d \times(\bar{e} \circ \overline{\mathbf{s}}))=i d,
$$

hold (in particular, by combining with the surjectivity of $\overline{\mathbf{s}}$ and $\overline{\mathbf{t}}$, one has $\overline{\mathbf{s}} \circ \bar{e}=i d, \overline{\mathbf{t}} \circ \bar{e}=i d$ on $M)$;

(v) (inverse) an isomorphism of differentiable stacks $\bar{i}: \mathcal{G} \rightarrow \mathcal{G}$ such that, up to 2-morphisms, the following identities

$$
\bar{m} \circ(\bar{i} \times i d \circ \Delta)=\bar{e} \circ \overline{\mathbf{s}}, \bar{m} \circ(i d \times \bar{i} \circ \Delta)=\bar{e} \circ \overline{\mathbf{t}},
$$

hold, where $\Delta$ is the diagonal map: $\mathcal{G} \rightarrow \mathcal{G} \times \mathcal{G}$.

Moreover, restricting to the identity section, the above 2-morphisms between maps are the $i d$ 2-morphisms. Namely, for example, the 2-morphism $\alpha$ induces the $i d$ 2-morphism between the following two maps:

$$
\bar{m} \circ((\bar{m} \circ(\bar{e} \times \bar{e} \circ \delta)) \times \bar{e} \circ \delta)=\bar{m} \circ(\bar{e} \times(\bar{m} \circ(\bar{e} \times \bar{e} \circ \delta)) \circ \delta),
$$

where $\delta$ is the diagonal map: $M \rightarrow M \times M$.

The terminology involving stacks in the above definition will be explained in detail in $\S 3$. For now, to get a general idea of these statements, one can take stacks simply to be manifolds.

Our main result is the following theorem.

Theorem 1.2 (Lie's third theorem). With each Weinstein groupoid one can associate a Lie algebroid. For every Lie algebroid $A$, there are naturally two Weinstein groupoids $\mathcal{G}(A)$ and $\mathcal{H}(A)$ with Lie algebroid $A$.

We can apply our result to the classical integrability problem, which studies exactly when a Lie algebroid can be integrated into a Lie groupoid.

Theorem 1.3. A Lie algebroid $A$ is integrable in the classical sense if and only if $\mathcal{H}(A)$ is representable, i.e. it is an honest (smooth) manifold. In this case $\mathcal{H}(A)$ is the source-simply connected Lie groupoid of $A$ (it is also called the Weinstein groupoid of $A$ in [CF03]).

We can also relate our work to the previous work on the integration of Lie algebroids via the following two theorems. 


\section{INTEGRATING LIE ALGEBROIDS VIA STACKS}

Theorem 1.4. Given a Weinstein groupoid $\mathcal{G}$, there is an associated local Lie groupoid $G_{\text {loc }}$ (canonical up to isomorphisms near the identity section) that has the same Lie algebroid as $\mathcal{G}$.

Theorem 1.5. The orbit spaces of $\mathcal{H}(A)$ and $\mathcal{G}(A)$ as topological spaces are both isomorphic to the topological groupoid of $A$ constructed in [CF03].

\section{Path spaces}

We define the $A_{0}$-path space, which is very similar to the $A$-paths defined in [CF03] (it is, in fact, a submanifold of the $A$-path space).

Definition 2.1. Given a Lie algebroid $A \stackrel{\pi}{\longrightarrow} M$ ( $A$ is assumed to be a Hausdorff manifold) with anchor $\rho: A \rightarrow T M$, a $C^{1}$ map $a: I=[0,1] \rightarrow A$ is an $A_{0}$-path if it satisfies the equation

$$
\rho(a(t))=\frac{d}{d t}(\pi \circ a(t))
$$

with boundary conditions $a(0)=0, a(1)=0, \dot{a}(0)=0, \dot{a}(1)=0$. We often denote the base path $\pi \circ a(t)$ in $M$ by $\gamma(t)$. We denote by $P_{0} A$ the set of all $A_{0}$-paths of $A$. It is a topological space with topology given by uniform convergence of maps. Omitting the boundary condition above, we get the definition of $A$-paths, and we denote the space of $A$-paths by $P_{a} A$.

We can equip $P_{0} A$ with the structure of a smooth (Banach) manifold modeled by $P \mathbb{R}^{n}=$ $C^{1}\left(I, \mathbb{R}^{n}\right)$ with norm $\|f\|^{2}=\sup \left\{|f|^{2}+\left|f^{\prime}\right|^{2}\right\} . P_{0} A$ is defined by equations on $P A$, so it inherits the structure of a Banach manifold from $P A$. See [CF03] and [Zhu04] for details.

Definition 2.2. Let $a(\epsilon, t)$ be a family of $A_{0}$-paths of class $C^{2}$ in $\epsilon$ and assume that their base paths $\gamma(\epsilon, t)$ have fixed end points. Let $\nabla$ be a connection on $A$ with torsion $T_{\nabla}$ defined as

$$
T_{\nabla}(\alpha, \beta)=\nabla_{\rho(\beta)} \alpha-\nabla_{\rho(\alpha)} \beta+[\alpha, \beta] .
$$

Then the solution $b=b(\epsilon, t)$ of the differential equation

$$
\partial_{t} b-\partial_{\epsilon} a=T_{\nabla}(a, b), \quad b(\epsilon, 0)=0
$$

does not depend on the choice of connection $\nabla$. Furthermore, $b(\cdot, t)$ is an $A$-path for every fixed $t$, i.e. $\rho(b(\epsilon, t))=(d / d \epsilon) \gamma(\epsilon, t)$. If the solution $b$ satisfies $b(\epsilon, 1)=0$, for all $\epsilon$, then $a_{0}$ and $a_{1}$ are said to be equivalent and we write $a_{0} \sim a_{1}$.

\section{Remark 1.}

(i) Here, $T_{\nabla}(a, b)$ is not quite well defined. We need to extend $a$ and $b$ by sections of $A, \alpha$ and $\beta$, such that $a(t)=\alpha(\gamma(t), t)$ and the same for $b$. Then $\left.T_{\nabla}(a, b)\right|_{\gamma(t)}:=\left.T_{\nabla}(\alpha, \beta)\right|_{\gamma(t)}$ at every point on the base path. However, the choice of extending sections does not affect the result.

(ii) A homotopy of $A$-paths [CF03] is defined by replacing $A_{0}$ by $A$ in the definition above. A similar result as above holds for $A$-paths [CF03]. So the above statement holds viewing $A_{0}$-paths as $A$-paths.

This flow of $A_{0}$-paths $a(\epsilon, t)$ generates a foliation $\mathcal{F}$. The $A_{0}$-path space is a Banach submanifold of the $A$-path space and $\mathcal{F}$ is the restricted foliation of the foliation defined in [CF03, $\S 4$ ]. For any foliation, there is an associated monodromy groupoid [MM03] (or fundamental groupoid as in [CW99]): the arrows are paths within a leaf up to homotopies with fixed end points inside the leaf. For any regular foliation on a smooth manifold its monodromy groupoid is a Lie groupoid in the sense of [CF03]. In our case, it is an infinite-dimensional groupoid equipped with a Banach manifold structure. Here, we slightly generalized the definition of Lie groupoids to the category of Banach manifolds by requiring the same conditions, but in the sense of Banach manifolds. Denote the 


\section{H.-H. Tseng And C. Zhu}

monodromy groupoid of $\mathcal{F}$ by $\operatorname{Mon}\left(P_{0} A\right) \underset{\mathbf{t}_{M}}{\stackrel{\mathbf{s}_{M}}{\rightrightarrows}} P_{0} A$. In a very similar way [MM03], one can also define the holonomy groupoid $\operatorname{Hol}(\mathcal{F})$ of $\mathcal{F}$ : the arrows are equivalence classes of paths with the same holonomy.

To obtain a finite-dimensional description we take an open cover $\left\{U_{i}\right\}_{i \in I}$ of $P_{0} A$ such that in each chart $U_{i}$ one can choose a transversal $P_{i}$ of the foliation $\mathcal{F}$. We can assume $I$ is countable as $P_{0} A$ is second countable. By [CF03, Proposition 4.8], each $P_{i}$ is a smooth manifold of dimension equal to that of $A$. Let $P=\coprod P_{i}$, which is a smooth immersed submanifold of $P_{0} A$. We can choose $\left\{U_{i}\right\}$ and transversal $\left\{P_{i}\right\}$ to satisfy the following conditions.

(i) If $U_{i}$ contains the constant path $0_{x}$ for some $x \in M$, then $U_{i}$ has the transversal $P_{i}$ containing all constant paths $0_{y}$ in $U_{i}$ for $y \in M$.

(ii) If $a(t) \in P_{i}$ for some $i$, then $a(1-t) \in P_{j}$ for some $j$.

It is possible to meet the above two conditions: for (i) we refer readers to [CF03, Proposition 4.8]. There the result is for $P_{a} A$. For $P_{0} A$, one has to use a smooth reparametrization $\tau$ with the properties:

(i) $\tau(t)=1$ for all $t \geqslant 1$ and $\tau(t)=0$ for all $t \leqslant 0$;

(ii) $\tau^{\prime}(t)>0$ for all $t \in(0,1)$.

Then $a^{\tau}(t):=\tau(t)^{\prime} a(\tau(t))$ is in $P_{0} A$ for all $a \in P_{a} A$. $\phi_{\tau}: a \mapsto a^{\tau}$ defines an injective bounded linear map from $P_{a} A \rightarrow P_{0} A$. Therefore, we can adapt the construction for $P_{a} A$ to our case by using $\phi_{\tau}$. For (ii), we define a map inv : $P_{0} A \rightarrow P_{0} A$ by $\operatorname{inv}(a(t))=a(1-t)$. Obviously inv is an isomorphism. In particular, it is open. So we can add $\operatorname{inv}\left(U_{i}\right)$ and $\operatorname{inv}\left(P_{i}\right)$ to the collection of open sets and transversals. The new collection will have the desired property.

The restriction $\left.\operatorname{Mon}\left(P_{0} A\right)\right|_{P}$ of $\operatorname{Mon}\left(P_{0} A\right)$ to $P$ is a finite-dimensional étale Lie groupoid (i.e. the source (hence, the target) map is a local diffeomorphism) [MRW87], which we denote by $\Gamma \rightrightarrows P$. For a different transversal $P^{\prime}$ the restriction of $\operatorname{Mon}\left(P_{0} A\right)$ to $P^{\prime}$ is another finite-dimensional étale Lie groupoid. All of these groupoids are related by 'Morita equivalence'. One can do the same to $\operatorname{Hol}\left(P_{0} A\right)$ and obtain a finite-dimensional étale Lie groupoid, which we denote by $\Gamma^{h} \underset{\mathbf{t}_{1}}{\stackrel{\mathbf{s}_{1}}{\rightrightarrows}} P$. Although these groupoids are Morita equivalent to each other, they are in general not Morita equivalent to the groupoids induced from $\operatorname{Mon}\left(P_{0} A\right)$.

We will build a Weinstein groupoid of $A$ based on this path space $P_{0} A$. One can interpret the 'identity section' as an embedding obtained from taking constant paths $0_{x}$, for all $x \in M$; the 'inverse' of a path $a(t)$ as $a(1-t)$; the 'source and target maps' $\mathbf{s}$ and $\mathbf{t}$ as taking the end points of the base path $\gamma(t)$. According to the two conditions above, these maps are also well defined on the finite-dimensional space $P$. As reparametrizations and projections are bounded linear operators in Banach space $C^{\infty}\left(I, \mathbb{R}^{n}\right)$, the maps defined above are smooth maps in $P_{0} A$, hence in $P$.

To define the multiplication, notice that for two $A$-paths $a_{1}, a_{0}$ in $P_{0} A$ such that the base paths satisfy $\gamma_{0}(1)=\gamma_{1}(0)$, one can define a 'concatenation' [CF03]:

$$
a_{1} \odot a_{0}= \begin{cases}2 a_{0}(2 t), & 0 \leqslant t \leqslant \frac{1}{2} \\ 2 a_{1}(2 t-1), & \frac{1}{2}<t \leqslant 1 .\end{cases}
$$

Concatenation is a bounded linear operator in the local charts, hence is a smooth map. However, it is not associative. Moreover, it is not well defined on $P$. If we quotient out by the equivalence relation induced by $\mathcal{F}$, concatenation is associative and well defined. However, after quotienting out by the equivalence, we may no longer end up with a smooth manifold. To overcome the difficulty, our solution is to pass to the world of differentiable stacks. 


\section{INTEGRATING LIE ALGEBROIDS VIA STACKS}

\section{Differentiable stacks and Lie groupoids}

The notion of stacks has been extensively studied in algebraic geometry for the past few decades (see, for example, [DM69, Vis89, LM00, BEFFGK]). Stacks can also be defined over other categories, such as the category of topological spaces and the category of smooth manifolds (see, for example, [AGV72, Pro96, Vis02, BX, Met03]). In this section we collect certain facts about stacks in the differentiable category that will be used in next sections. Detailed treatments of these can be found in the literature (see, for example, [Pro96, BX, Met03]). Some recent application of differentiable stacks can be found in [BX03].

\subsection{Definitions}

Let $\mathcal{C}$ be the category of differentiable manifolds (second countable but not necessarily Hausdorff). A stack over $\mathcal{C}$ is a category fibred in groupoids satisfying two conditions: 'isomorphism is a sheaf', and 'descent datum is effective', see [BX] and [Met03] for the complete definition.

A manifold is a stack over $\mathcal{C}$. We call such stacks representable. Morphisms between stacks are just functors between fibred categories. A morphism $f: \mathcal{X} \rightarrow \mathcal{Y}$ is a representable submersion if for any morphism $M \rightarrow \mathcal{Y}$ from a manifold $M$, the fiber product $\mathcal{X} \times \mathcal{Y} M$ is representable and the induced morphism $\mathcal{X} \times \mathcal{Y} M \rightarrow M$ is a submersion (between manifolds). If, in addition, $\mathcal{X} \times \mathcal{Y} M \rightarrow M$ is surjective, then $f$ is called a representable surjective submersion [BX].

A differentiable stack is a stack $\mathcal{X}$ over $\mathcal{C}$ together with a representable surjective submersion $\pi: X \rightarrow \mathcal{X}$ from a Hausdorff smooth manifold $X$ (called an atlas). A manifold is a differentiable stack by identifying it with its functor of points. For a Lie group $G$, the category of principal $G$-bundles is an example of a differentiable stack.

Properties of morphisms between differentiable stacks can be defined by considering pullbacks to atlases. In this way one can define what it means for a morphism to be smooth, étale, an immersion, a closed immersion and an injective immersion; see, for instance, [BX] and [Met03]. To check whether a morphism has these properties it suffices to check on a particular atlas. Compositions of representable (surjective) submersions are still representable (surjective) submersion. Representable (surjective) submersions are stable under base-change.

A differentiable stack $\mathcal{X}$ is called étale if it has an atlas $\pi: X \rightarrow \mathcal{X}$ such that $\pi$ is étale.

\subsection{Stacks and groupoids}

One can go between differentiable stacks and Lie groupoids. For a differentiable stack $\mathcal{X}$ with an atlas $X_{0} \rightarrow \mathcal{X}$, there is a Lie groupoid $X_{1}:=X_{0} \times \mathcal{X} X_{0} \rightrightarrows X_{0}$ with the two maps being projections. This groupoid is called a groupoid presentation of $\mathcal{X}$. An étale differentiable stack can be presented by an étale groupoid. Given a Lie groupoid $G=\left(G_{1} \rightrightarrows G_{0}\right)$, the category of principal $G$ bundles $B G$ is a differentiable stack with an atlas $G_{0} \rightarrow B G$ such that $G_{1}=G_{0} \times_{B G} G_{0}$. See [Vis89, Pro96, BX, Met03] for details.

There are some ambiguities: Different atlases give different groupoids, and two Lie groupoids may represent the same stack. The following result clarifies this.

Proposition 3.1 (see [Pro96, BX, Met03]). Two Lie groupoids present isomorphic differential stacks if and only if they are Morita equivalent.

In other words, differentiable stacks correspond to Morita equivalence classes of Lie groupoids. Also, smooth 1-morphisms between differentiable stacks correspond to Hilsum-Skandalis (HS) morphisms given by one-side principal bibundles of the groupoids, see [Pro96] for details. 2-morphisms of differentiable stacks correspond to 2-morphisms of Lie groupoids (see [Pro96] and [Met03] for a definition). 


\section{H.-H. Tseng And C. Zhu}

\subsection{Fibre products and submersions}

It is convenient to use invariant maps to produce maps between stacks. In this section we state some results concerning invariant maps and fiber products which will be used later in the construction of the Weinstein groupoids. The proofs are standard and can be found in [Zhu04].

Lemma 3.2. Given a Lie groupoid $G:=\left(G_{1} \rightrightarrows G_{0}\right)$ and a manifold $M$, any $G$-invariant map $f: G_{0} \rightarrow M$ induces a morphism between differentiable stacks $\bar{f}: B G \rightarrow M$ such that $f=\bar{f} \circ \phi$, where $\phi: G_{0} \rightarrow B G$ is the covering map of atlases.

Definition 3.3 ((Surjective) submersions). A morphism $f: \mathcal{X} \rightarrow \mathcal{Y}$ of differentiable stacks is called a submersion if for any atlas $M \rightarrow \mathcal{X}$, the composition $M \rightarrow \mathcal{X} \rightarrow \mathcal{Y}$ satisfies the following: for any atlas $N \rightarrow \mathcal{Y}$ the induced morphism $M \times \mathcal{Y} N \rightarrow N$ is a submersion. A surjective submersion is a submersion that is also an epimorphism.

Note that this definition is different from that in [Met03]. A representable submersion is a submersion, but the converse is not true.

Proposition 3.4 (Fibred products). Let $Z$ be a manifold and $f: \mathcal{X} \rightarrow Z$ and $g: \mathcal{Y} \rightarrow Z$ be morphisms of differentiable stacks. If either $f$ or $g$ is a submersion, then $\mathcal{X} \times_{Z} \mathcal{Y}$ is a differentiable stack. Moreover, let $X \rightarrow \mathcal{X}, Y \rightarrow \mathcal{Y}$ be atlases for $\mathcal{X}$ and $\mathcal{Y}$, respectively. Then $X \times{ }_{Z} Y \rightarrow \mathcal{X} \times{ }_{Z} \mathcal{Y}$ is an atlas for $\mathcal{X} \times_{Z} \mathcal{Y}$. Moreover, put $X_{1}=X \times \mathcal{X} X$ and $Y_{1}=Y \times \mathcal{Y} Y$, then $\mathcal{X} \times_{Z} \mathcal{Y}$ is presented by the groupoid $\left(X_{1} \times{ }_{Z} Y_{1} \rightrightarrows X \times{ }_{Z} Y\right)$.

Lemma 3.5. If a $G$-invariant map $f: G_{0} \rightarrow M$ is a submersion, then the induced map $\bar{f}: B G \rightarrow M$ is a submersion of differentiable stacks.

It is not hard to see that the construction of stacks in the category of smooth manifolds can be extended to the category of Banach manifolds, yielding the notion of Banach stacks. Many properties of differentiable stacks, including those discussed here, are also shared by Banach stacks. Also, the 2-category of differentiable stacks can be obtained from the 2-category of Banach stacks by restricting the base category.

\section{The Weinstein groupoids of Lie algebroids}

\subsection{The construction}

Recall that in $\S 2.1$, given a Lie algebroid $A$, we constructed an étale groupoid $\Gamma \rightrightarrows P$. We obtain an étale differential stack $\mathcal{G}(A)$ presented by $\Gamma \rightrightarrows P$. For a different transversal $P^{\prime}$, the restriction $\Gamma^{\prime}=$ $\left.\operatorname{Mon}\left(P_{0} A\right)\right|_{P^{\prime}}$ is Morita equivalent to $\Gamma$ through the finite-dimensional bibundle $\mathbf{s}_{M}^{-1}(P) \cap \mathbf{t}_{M}^{-1}\left(P^{\prime}\right)$. So they represent isomorphic differential stacks. Therefore, we might base our discussion on $\Gamma \rightrightarrows P$. As $\operatorname{Mon}\left(P_{0} A\right) \rightrightarrows P_{0} A$ is Morita equivalent to $\Gamma \rightrightarrows P$ through the Banach bibundle $\mathbf{s}_{M}^{-1}(P), \mathcal{G}(A)$ can also be presented by $\operatorname{Mon}\left(P_{0} A\right)$ as a Banach stack.

In this section, we construct two Weinstein groupoids $\mathcal{G}(A)$ and $\mathcal{H}(A)$ for every Lie algebroid $A$ and prove Theorem 1.3.

We begin with $\mathcal{G}(A)$. We first define the inverse, identity section, source and target maps on the level of groupoids.

Definition 4.1. Define the following.

- $i:(\Gamma \rightrightarrows P) \rightarrow(\Gamma \rightrightarrows P)$ by $g=[a(\epsilon, t)] \mapsto[a(\epsilon, 1-t)]$, where [·] denotes the homotopy class in $\operatorname{Mon}\left(P_{0} A\right)$.

- $e: M \rightarrow(\Gamma \rightrightarrows P)$ by $x \mapsto 1_{0_{x}}$, where $1_{0_{x}}$ denotes the identity homotopy of the constant path $0_{x}$. 


\section{INTEGRATING LIE ALGEBROIDS VIA STACKS}

- $\mathbf{s}:(\Gamma \rightrightarrows P) \rightarrow M$ by $g=[a(\epsilon, t)] \mapsto \gamma(0,0)(=\gamma(\epsilon, 0), \forall \epsilon)$, where $\gamma$ is the base path of $a$.

- $\mathbf{t}:(\Gamma \rightrightarrows P) \rightarrow M$ by $g=[a(\epsilon, t)] \mapsto \gamma(0,1)(=\gamma(\epsilon, 1), \forall \epsilon)$.

These maps can be defined similarly on $\operatorname{Mon}\left(P_{0} A\right) \rightrightarrows P_{0} A$. These maps are all bounded linear maps in the local charts of $\operatorname{Mon}\left(P_{0} A\right)$. Therefore, they are smooth homomorphisms between Lie groupoids. Hence, they defined smooth morphisms between differentiable stacks. We denote the maps corresponding to $i, e, \mathbf{s}$ and $\mathbf{t}$ on the stack level by $\bar{i}, \bar{e}, \overline{\mathbf{s}}$ and $\overline{\mathbf{t}}$, respectively.

Lemma 4.2. The maps $\overline{\mathbf{s}}$ and $\overline{\mathbf{t}}$ are surjective submersions. The map $\bar{e}: M \rightarrow \mathcal{G}(A)$ is an injective immersion. The map $\bar{i}$ is an isomorphism.

Proof. As any path through $x$ in $M$ can be lifted to a path in $P$ passing through any given preimage of $x, \mathbf{s}$ and $\mathbf{t}$ restricted to $P$ are $\Gamma$-invariant and submersions. According to Lemmas 3.2 and 3.5, the induced maps $\overline{\mathbf{s}}$ and $\overline{\mathbf{t}}$ are submersions.

Denote by $e_{0}$ the restricted map of $e$ on the level of objects: $e_{0}: M \rightarrow P$. Note that $e_{0}$ fits into the following diagram (which is not commutative).

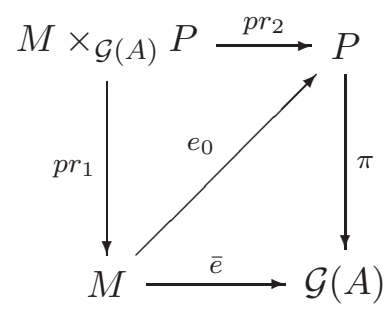

Consider $x=(f: U \rightarrow M) \in M, \bar{e}(x)=U \times_{e_{0} \circ f, G_{0}} G_{1}$ as a $G$-torsor, and $e_{0}(x)=\left(e_{0} \circ f\right.$ : $\left.U \rightarrow G_{0}\right) \in G_{0}$. Consider also $y=\left(g: U \rightarrow G_{0}\right) \in G_{0}, \pi(y)=U \times_{g, G_{0}} G_{1}$. A typical object of $M_{i} \times{ }_{\mathcal{G}} G_{0}$ is $(x, \eta, y)$ where $\eta$ is a morphism of $G$-torsors from $\bar{e}(x)$ to $\pi(y)$ over $i d_{U}$ of $U$. Then by the equivariancy of $\eta$, we have a map $\phi: U \rightarrow G_{1}$, such that $e_{0} \circ f=g \cdot \phi$. Therefore, we have a map $\alpha: M \times_{\mathcal{G}(A)} G_{0} \rightarrow G_{1}$ given by $\alpha(x, \eta, y)=\phi$, such that

$$
e_{0} \circ p r_{1}=p r_{2} \cdot \alpha .
$$

As $\pi$ is étale, so is $p r_{1}$. Moreover, as $e_{0}$ is an embedding, $p r_{2}$ must be an immersion. This shows that $\bar{e}$ is an immersion for one atlas, hence $\bar{e}$ is an immersion.

As $\mathbf{s} \circ e=\mathbf{t} \circ e=i d$ on the level of groupoids, the same identity passes to identity on the level of differentiable stacks. As $\overline{\mathbf{s}} \circ \bar{e}=\overline{\mathbf{t}} \circ \bar{e}=i d$, it is easy to see that $\bar{e}$ must be monomorphic and $\overline{\mathbf{s}}$ (and $\overline{\mathbf{t}}$ ) must be epimorphic.

The map $i$ is an isomorphism of groupoids, hence it induces an isomorphism at the level of stacks.

Now we define the multiplication in the infinite-dimensional presentation. First we extend 'concatenation' to $\operatorname{Mon}\left(P_{0} A\right)$. Consider two elements $g_{1}, g_{0} \in \operatorname{Mon}\left(P_{0} A\right)$ whose base paths on $M$ are connected at the end points. Suppose $g_{i}$ is represented by $a_{i}(\epsilon, t)$. Define

$$
g_{1} \odot g_{0}=\left[a_{1}(\epsilon, t) \odot_{t} a_{0}(\epsilon, t)\right],
$$

where $\odot_{t}$ means concatenation with respect to the parameter $t$ and the $[\cdot]$ denotes the equivalence class of homotopies.

Note that $\mathbf{s} \circ \mathbf{s}_{M}=\mathbf{s} \circ \mathbf{t}_{M}$ and $\mathbf{t} \circ \mathbf{s}_{M}=\mathbf{t} \circ \mathbf{t}_{M}$ are surjective submersions by reasoning similar to that in the above, where $\mathbf{t}_{M}$ and $\mathbf{s}_{M}$ are source and target maps of $\operatorname{Mon}\left(P_{0} A\right) \rightrightarrows P_{0} A$. Hence, by Proposition 3.4,

$$
\operatorname{Mon}\left(P_{0} A\right) \times_{\mathbf{s o s}_{M}, M, \text { tot }_{M}} \operatorname{Mon}\left(P_{0} A\right) \rightrightarrows P_{0} A
$$




\section{H.-H. Tseng And C. Zhu}

with source and target maps $\mathbf{s}_{M} \times \mathbf{s}_{M}$ and $\mathbf{t}_{M} \times \mathbf{t}_{M}$ is a Lie groupoid and it presents the stack $\mathcal{G} \times_{\overline{\mathbf{s}}, M, \overline{\mathbf{t}}} \mathcal{G}$.

Finally, let $m$ be the following smooth homomorphism between Lie groupoids.

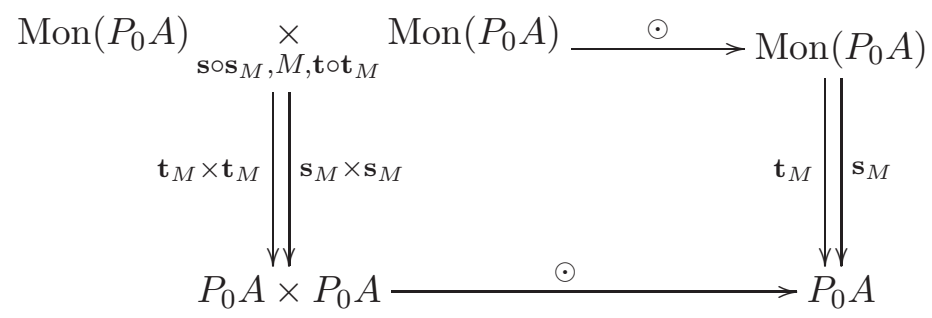

Multiplication is less obvious for the étale presentation $\Gamma \rightrightarrows P$. We will have to define the multiplication through a HS morphism.

Viewing $P$ as a submanifold of $P_{0} A$, let $E=\mathbf{s}_{M}^{-1}(P) \cap \mathbf{t}_{M}^{-1}\left(m\left(P \times_{M} P\right)\right) \subset \operatorname{Mon}\left(P_{0} A\right)$. As $\mathbf{s}_{M}$ and $\mathbf{t}_{M}$ are surjective submersions and $m\left(P \times_{M} P\right) \cong P \times_{M} P$ is a submanifold of $P_{0} A, E$ is a smooth manifold. As $P$ is a transversal, $\mathbf{t}_{M}: E \rightarrow m\left(P \times_{M} P\right)$ is étale. Moreover, $\operatorname{dim} m\left(P \times_{M} P\right)=$ $2 \operatorname{dim} P-\operatorname{dim} M$. So $E$ is finite dimensional. Further, note that $m: P_{0} A \times P_{0} A \rightarrow P_{0} A$ is injective and its 'inverse' $m^{-1}$ defined on the image of $m$ is given by

$$
m^{-1}: b(t) \mapsto\left(b\left(2 t_{1}\right), b\left(1-2 t_{2}\right)\right), \quad t_{1} \in\left[0, \frac{1}{2}\right], \quad t_{2} \in\left[\frac{1}{2}, 1\right],
$$

which is bounded linear in a local chart. Let $\pi_{1}=m^{-1} \circ \mathbf{t}_{M}: E \rightarrow P \times_{M} P$ and $\pi_{2}=\mathbf{s}_{M}: E \rightarrow P$. Then it is routine to check that the bibundle $\left(E, \pi_{1}, \pi_{2}\right)$ gives a HS morphism from $\Gamma \times{ }_{M} \Gamma \rightrightarrows P \times_{M} P$ to $\Gamma \rightrightarrows P$. It is not hard to verify that on the level of stacks $\left(E, \pi_{1}, \pi_{2}\right)$ and $m$ give two 1-morphisms differed by a 2-morphism. Thus, after modifying $E$ by this 2-morphism, we get another HS morphism $\left(E_{m}, \pi_{1}^{\prime}, \pi_{2}^{\prime}\right)$, which presents the same map as $m$. Moreover, $E_{m} \cong E$ as bibundles.

Therefore, we have the following definition.

Definition 4.3. Define $\bar{m}: \mathcal{G}(A) \times_{\overline{\mathbf{s}}, \overline{\mathbf{t}}} \mathcal{G}(A) \rightarrow \mathcal{G}(A)$ to be the smooth morphism between étale stacks presented by $\left(E_{m}, \pi_{1}^{\prime}, \pi_{2}^{\prime}\right)$.

Remark 2. If we use $\operatorname{Mon}\left(P_{0} A\right)$ as the presentation, $\bar{m}$ is also presented by $m$.

Lemma 4.4. The multiplication $\bar{m}: \mathcal{G}(A) \times \mathcal{G}(A) \rightarrow \mathcal{G}(A)$ is a smooth morphism between étale stacks and is associative up to a 2-morphism. That is, the diagram

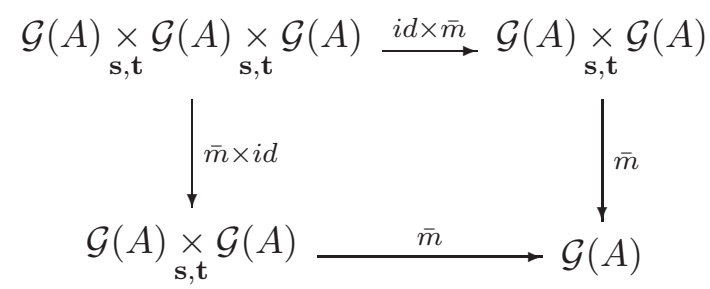

is 2-commutative, i.e. there exists a 2-morphism $\alpha: \bar{m} \circ(\bar{m} \times i d) \rightarrow \bar{m} \circ(i d \times \bar{m})$.

Before the proof, we give a general remark about 2-morphisms.

Remark 3. For two groupoid homomorphisms $f$ and $g$ between $G$ and $H$, a 2-morphism between the induced maps on the level of stacks represented by $f$ to $g$ is just a smooth map $\alpha: G_{0} \rightarrow H_{1}$ such that $\alpha(\gamma x)=g(\gamma) \alpha(x) f(\gamma)^{-1}$, where $x \in G_{0}$ and $\gamma \in G_{1}$. This in particular gives us $f(x)=g(x) \cdot \alpha(x)$. So it is easy to see that not every two morphisms can be connected by a 2-morphism and when they do, the 2-morphism may not be unique (for example, this happens when the isotropy group is non-trivial and abelian). 


\section{INTEGRATING LIE ALGEBROIDS VIA STACKS}

Proof. We will establish the 2-morphism on the level of Banach stacks. Note that a smooth morphism in the category of Banach manifolds between finite-dimensional manifolds is a smooth morphism in the category of finite-dimensional smooth manifolds. Therefore, the 2-morphism we will establish gives a 2-morphism for the étale stacks.

Take the Banach presentation $\operatorname{Mon}\left(P_{0} A\right)$, then $\bar{m}$ can simply be presented as a homomorphism between groupoids as in (4.1). According to Remark 3, we now construct a 2-morphism $\alpha: P_{0} A \times_{M}$ $P_{0} A \times_{M} P_{0} A \rightarrow \operatorname{Mon}\left(P_{0} A\right)$ in the following diagram.

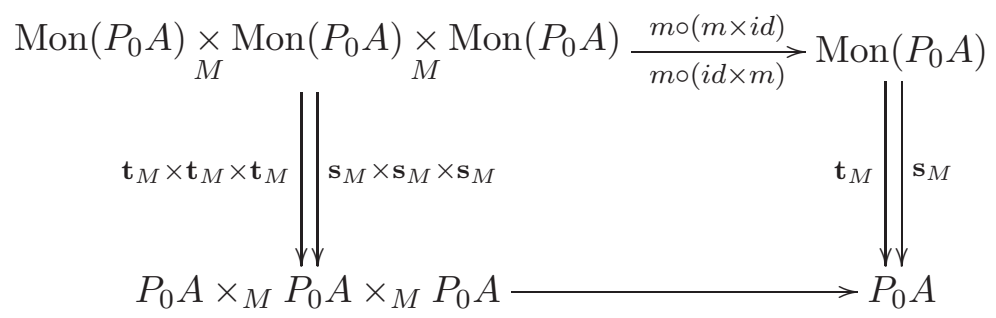

Let $\alpha\left(a_{1}, a_{2}, a_{3}\right)$ be the natural rescaling between $a_{1} \odot\left(a_{2} \odot a_{3}\right)$ and $\left(a_{1} \odot a_{2}\right) \odot a_{3}$. Namely, $\alpha\left(a_{1}, a_{2}, a_{3}\right)$ is the homotopy class represented by

$$
a(\epsilon, t)=\left((1-\epsilon)+\epsilon \sigma^{\prime}(t)\right) a((1-\epsilon) t+\epsilon \sigma(t)),
$$

where $\sigma(t)$ is a smooth reparametrization such that $\sigma(1 / 4)=1 / 2, \sigma(1 / 2)=3 / 4$. In local charts, $\alpha$ is a bounded linear operator. Therefore, it is a smooth morphism between Banach spaces. Moreover, for $x \in P_{0} A \times_{M} P_{0} A \times_{M} P_{0} A$ and $g \in \operatorname{Mon}\left(P_{0} A\right) \times_{M} \operatorname{Mon}\left(P_{0} A\right) \times_{M} \operatorname{Mon}\left(P_{0} A\right), \alpha(g \cdot x)=$ $m \circ(i d \times m)(g) \cdot \alpha(x) \cdot(m \circ(m \times i d))^{-1}(g)$. In fact, it is not hard to see $m \circ(m \times i d)(x)=$ $m \circ(i d \times m)(x) \cdot \alpha(x)$. Counting homotopy inside and noting that we quotient out the homotopies of homotopies, the former equation is also true. Therefore, $\alpha$ serves as the desired 2-morphism.

One might be curious about whether there are further obstructions to associativity. There are six ways to multiply four elements in $\mathcal{G}(A)$. Put

$$
\begin{aligned}
& F_{1}=\bar{m} \circ \bar{m} \times i d \circ \bar{m} \times i d \times i d, \\
& F_{2}=\bar{m} \circ i d \times \bar{m} \circ \bar{m} \times i d \times i d, \\
& F_{3}=\bar{m} \circ \bar{m} \times i d \circ i d \times i d \times \bar{m}, \\
& F_{4}=\bar{m} \circ i d \times \bar{m} \circ i d \times i d \times \bar{m}, \\
& F_{5}=\bar{m} \circ i d \times \bar{m} \circ i d \times \bar{m} \times i d, \\
& F_{6}=\bar{m} \circ \bar{m} \times i d \circ i d \times \bar{m} \times i d .
\end{aligned}
$$

These morphisms fit into the following commutative cube.

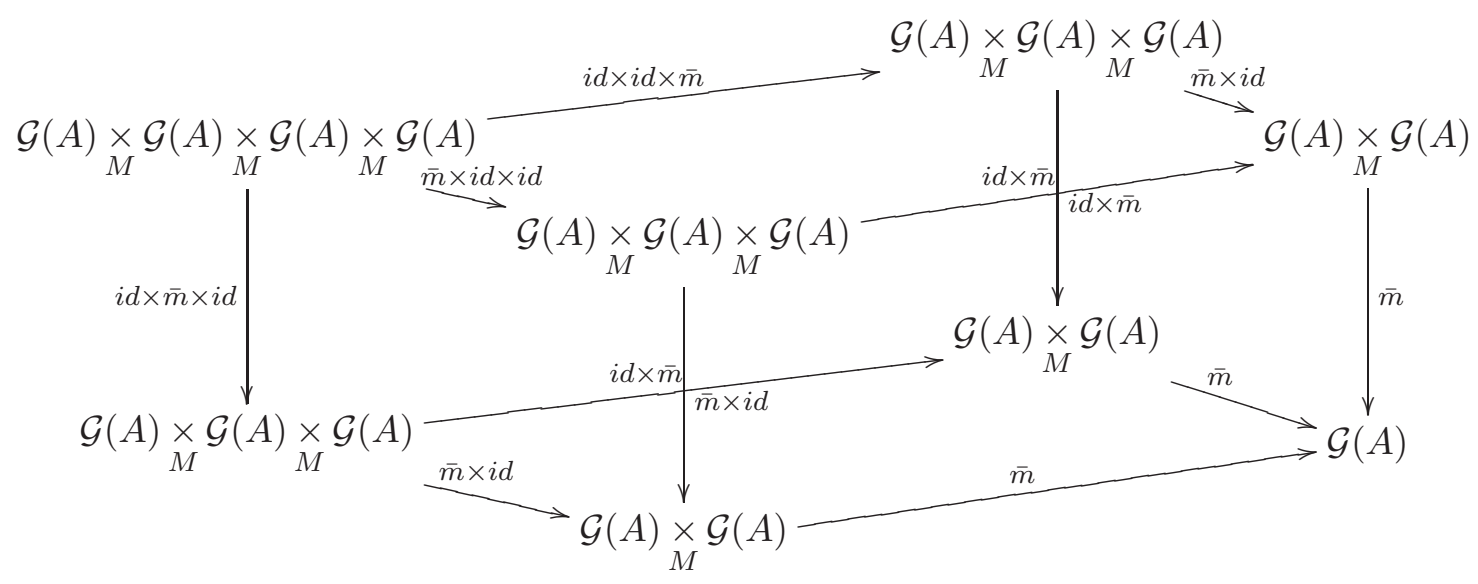




\section{H.-H. Tseng And C. Zhu}

There is a 2-morphism on each face of the cube to connect $F_{i}$ and $F_{i+1}\left(F_{7}=F_{1}\right)$, constructed as in the last lemma. Let $\alpha_{i}: F_{i} \rightarrow F_{i+1}$. Will the composition $\alpha_{6} \circ \alpha_{6} \circ \cdots \circ \alpha_{1}$ be the identity 2-morphism? If so, given any two different ways of multiplying four (hence any number of) elements, different methods to obtain 2-morphisms between them will give rise to the same 2-morphism. As 2-morphisms between two 1-morphisms are not unique if our differential stacks are not honest manifolds, it is necessary to study the existence of further obstructions.

Proposition 4.5. There is no further obstruction to associativity of $\bar{m}$ in $\mathcal{G}(A)$.

Proof. In the presentation $\operatorname{Mon}\left(P_{0} A\right)$ of $\mathcal{G}(A)$, the $\alpha_{i}$ constructed above can be explicitly expressed as a smooth morphism: $P_{0} A \times_{M} P_{0} A \times_{M} P_{0} A \times_{M} P_{0} A \rightarrow \operatorname{Mon}\left(P_{0} A\right)$. More precisely, according to the lemma above, $\alpha_{i}\left(a_{1}, a_{2}, a_{3}, a_{4}\right)$ is the natural rescaling between $F_{i}\left(a_{1}, a_{2}, a_{3}, a_{4}\right)$ and $F_{i+1}\left(a_{1}, a_{2}, a_{3}, a_{4}\right)$. Here, by abuse of notation, we also denote the homomorphism on the groupoid level by $F_{i}$. It is not hard to see that $\alpha_{6} \circ \alpha_{5} \circ \cdots \circ \alpha_{1}$ is represented by a rescaling that is homotopic to the identity homotopy between $A_{0}$-paths.

Therefore, the composed 2-morphism is actually identity as $\operatorname{Mon}\left(P_{0} A\right)$ is made up by the homotopy of homotopy of $A_{0}$-paths. We also note that identity morphism in the category of Banach manifolds between two finite-dimensional manifolds is identity morphism in the category of finite-dimensional smooth manifolds. Therefore, there is no further obstructions even for 2-morphisms of étale stacks.

Now to show $\mathcal{G}(A)$ is a Weinstein groupoid, it remains to show that the identities in items $(4)$ and (5) in Definition 1.1 hold and the 2-morphisms in these identities are identity 2-morphisms when restricted to $M$. Note that for any $A_{0}$-path $a(t)$, we have

$$
a(t) \odot_{t} 1_{\gamma(0)} \sim a(t), \quad a(1-t) \odot_{t} a(t) \sim \gamma(0),
$$

where $\gamma$ is the base path of $a(t)$. Using Remark 3(i), we can see that on the groupoid level $m \circ$ $((e \circ \mathbf{t}) \times i d)$ and $i d$ only differ by a 2-morphism, and the same for the pairs $m \circ(i \times i d)$ and $e \circ \mathbf{s}$, $\mathbf{s} \circ m$ and $\mathbf{s} \circ p r_{1}$. Therefore the corresponding identities hold on the level of differentiable stacks. Transform them to stacks and the rest of the identities also follow. Moreover, the 2-morphisms (in all presentations of $\mathcal{G}(A)$ we have described above) are formed by rescalings. When they restrict to constant paths in $M$, they are just $i d$.

Summing up what we have discussed above, $\mathcal{G}(A)$ with all the structures we have given is a Weinstein groupoid over $M$.

We further comment that one can construct another natural Weinstein groupoid $\mathcal{H}(A)$ associated with $A$ exactly in the same way as $\mathcal{G}(A)$ by the Lie groupoid $\operatorname{Hol}\left(P_{0} A\right)$ or $\Gamma^{h} \underset{\mathbf{t}_{1}}{\stackrel{\mathbf{s}_{1}}{\rightrightarrows}} P$ as they are Morita equivalent by a similar reason as their monodromy counterparts. One can establish the identity section, the inverse, etc., even the multiplication in exactly the same way. One only has to note that in the construction of the multiplication, the 2-morphism in the associativity diagram is the holonomy class (instead of homotopy class) of the reparametrization (3). One can do so because homotopic paths have the same holonomy. Moreover, by the same reason, there is no further obstructions to the multiplication on $\mathcal{H}(A)$.

Finally, we want to comment about the Hausdorffness of the source fibres (hence, the target fibres by the inverse) of $\mathcal{G}(A)$ and $\mathcal{H}(A)$.

Definition 4.6. An étale differentiable stack $\mathcal{X}$ is Hausdorff if and only if the diagonal map

$$
\Delta: \mathcal{X} \rightarrow \mathcal{X} \times \mathcal{X}
$$

is a closed immersion. 


\section{INTEGRATING LIE ALGEBROIDS VIA STACKS}

Remark 4 . In the case when $\mathcal{X}$ is a manifold, the diagonal map being a closed immersion is equivalent to its image being closed. Hence, this notion coincides with the usual Hausdorffness for manifolds.

Unlike the case of Lie groupoids, the source fibre of $\mathcal{G}(A)$ or $\mathcal{H}(A)$ is not, in general, Hausdorff. (see Example 1). The obstruction lies inside the foliation $\mathcal{F}$ defined in $\S 2.1$.

Proposition 4.7. The source fibre of $\mathcal{G}(A)$ is Hausdorff if and only if the leaves of the foliation $\mathcal{F}$ are closed. The same is true for $\mathcal{H}(A)$.

Proof. We prove this for $\mathcal{G}(A)$. The proof for $\mathcal{H}(A)$ is similar. Let $P$ be the étale atlas we have chosen. Then the source fibre $\overline{\mathbf{s}}^{-1}(x)=x \times_{M, \overline{\mathbf{s}}} \mathcal{G}(A)$ is a differentiable stack presented by $\mathbf{s}^{-1}(x)$ by Proposition 3.4. Consider the following diagram.

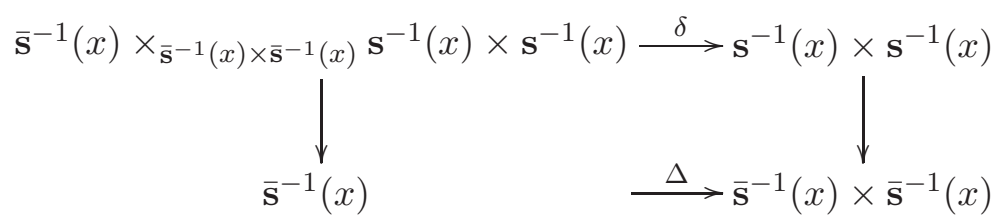

As $\Gamma=P \times_{\mathcal{G}(A)} P$, it is not hard to check that $\overline{\mathbf{s}}^{-1}(x) \times_{\overline{\mathbf{s}}^{-1}(x) \times \overline{\mathbf{s}}^{-1}(x)} \mathbf{s}^{-1}(x) \times \mathbf{s}^{-1}(x)$ is isomorphic to $\left.\Gamma\right|_{\mathbf{s}^{-1}(x)}$ and $\delta$ is just $\mathbf{s}_{1} \times \mathbf{t}_{1}$, where $\mathbf{s}_{1}$ and $\mathbf{t}_{1}$ are the source and target maps of $\Gamma$. Obviously $\mathbf{s}_{1} \times \mathbf{t}_{1}$ is an immersion as $\Gamma$ is an étale groupoid. Moreover, the image of $\delta$ is closed by the following argument: take a convergent sequence $\left(a_{0}^{i}(t), a_{1}^{i}(t)\right)$ of $A_{0}$-path with the limit $\left(a_{0}(t), a_{1}(t)\right)$. Suppose that $\left(a_{0}^{i}(t), a_{1}^{i}(t)\right)$ is inside the image of $\delta$, i.e. $a_{0}^{i}(t) \sim a_{1}^{i}(t)$. Let $\bar{a}$ denote the inverse path of $a$, we have $\bar{a}_{0}^{i}(t) \odot a_{1}^{i}(t) \sim 1_{x}$, i.e. they stay in the same leaf of the foliation $\mathcal{F}$. Hence, the limit path $\bar{a}_{0}(t) \odot a_{1}(t) \sim 1_{x}$ (i.e. $\left(a_{0}(t), a_{1}(t)\right)$ is also inside the image of $\delta$ ) if and only if the leaves of $\mathcal{F}$ are closed.

Example 1 (Non-Hausdorff source fibres). Let $M$ be $S^{2} \times S^{2}$ with 2 -form $\Omega=(\omega, \mu \omega)$. Let the Lie algebroid $A$ over $M$ be $T M \times \mathbb{R}$ with Lie bracket

$$
[(V, f),(W, g)]=\left([V, W], L_{V}(g)-L_{W}(h)+\Omega(V, W)\right)
$$

and anchor the projection onto TM (see [CW99, ch. 16] or [AM84]). Let $(a(\epsilon, t), u(\epsilon, t))$ be an $A_{0}$-homotopy, where the first component is in $T M$ and the second component is in the trivial bundle $\mathbb{R}$. The condition of being an $A_{0}$-path here is equivalent to $a=(d / d t) \gamma$ and boundary conditions, where $\gamma$ is the base path. Moreover, the first component of (1) is the usual $A_{0}$-homotopy equation for $T M$, which simply induces the homotopy of the base paths. The second component of (1) is

$$
\partial_{t} v-\partial_{\epsilon} u=\Omega(a, b)
$$

where $b$ in $(1)$ is $(b, v)$ above. Hence, $b=(d / d \epsilon) \gamma$. Integrating the above equation and using the boundary condition of $v$, we have

$$
\int_{0}^{1} u(0, t) d t-\int_{0}^{1} u(1, t) d t=\int_{\gamma} \Omega
$$

Let the period group $\Lambda$ of $\Omega$ at a point $x \in M$ be

$$
\Lambda_{x}=\int_{\gamma} \Omega, \quad[\gamma] \in \pi_{2}(M, x) .
$$

As $M$ is simply connected, one can actually show that $(\gamma(0, t), u(0, t)) \sim(\gamma(1, t), u(1, t))$ if and only if $\gamma(0, t)$ and $\gamma(1, t)$ have the same end points and $\int_{0}^{1}\left(u_{0}-u_{1}\right) d t \in \Lambda$. Then, in the case when $\mu$ is irrational, $\Lambda_{x}$ is dense in $\mathbb{R}$ for all $x$. Hence, there exist sequences $u_{0}^{i} \rightarrow u_{0}$ and $u_{1}^{i} \rightarrow u_{1}$ such that $\int_{0}^{1}\left(u_{0}^{i}-u_{1}^{i}\right) \in \Lambda$ but the limit $\int_{0}^{1}\left(u_{0}-u_{1}\right) \notin \Lambda$. Hence the leaves of the foliation $\mathcal{F}$ are not closed. 


\section{H.-H. Tseng AND C. Zhu}

In particular the $\mathcal{G}(A)$ and $\mathcal{H}(A)$ are not Lie groupoids. In the case when $\mu$ is rational, $\Lambda$ is discrete. In fact, the Lie algebroid $T M \times \mathbb{R}$ is isomorphic to the Lie algebroid associated to $M$ viewed as a Jacobi manifold. As in [CZ04], $\mathcal{G}(A)$ and $\mathcal{H}(A)$ are both the Lie groupoid $\left(S^{3} \otimes S^{3}\right) \otimes\left(S^{3} \otimes S^{3}\right)$, where $\otimes$ denotes the tensor product of $S^{1}$ bundles. Therefore, by varying $\mu \in \mathbb{R}$ we obtain a series of Weinstein groupoids which are only Lie groupoids for a measure 0 set in $\mathbb{R}$.

\subsection{The Integrability of Lie algebroids}

The integrability of $A$ and the representability of $\mathcal{G}(A)$ are not exactly the same, due to the presence of isotropy groups. However, as holonomy groupoids are always effective [MM03], we will show that the integrability of $A$ is equivalent to the representability of $\mathcal{H}(A)$.

Definition 4.8 (Orbit spaces). Let $\mathcal{X}$ be a differentiable stack presented by a Lie groupoid $X=$ $\left(X_{1} \rightrightarrows X_{0}\right)$. The orbit space of $\mathcal{X}$ is defined as the topological quotient $X_{0} / X_{1}$. Throughout the paper, when we mention the orbit space is a smooth manifold, we mean it has the natural smooth manifold structure induced from $X_{0}$ (i.e. the projection $X_{0} \rightarrow X_{0} / X_{1}$ is smooth).

Proof. We have to show the topological quotient is independent of choice of presentations. Suppose that there is another presentation $Y$ that is Morita equivalent to $X$ through $\left(E, J_{X}, J_{Y}\right)$. Let $O_{x}$ be the orbit of $X_{1}$ in $X_{0}$ through point $x$. By the fact that both groupoid actions are free and transitive fiber-wise, $J_{Y} \circ J_{X}^{-1}\left(O_{x}\right)$ is another orbit $O_{y}$ of $Y$. In this way, there is a one-to-one correspondence between orbits of $X$ and $Y$. Hence, $Y_{0} / Y_{1}$ understood as the space of orbits is the same as $X_{0} / X_{1}$.

Theorem 4.9. A Lie algebroid $A$ is integrable in the classical sense, i.e. there is a Lie groupoid whose Lie algebroid is $A$, if and only if the orbit space of $\mathcal{G}(A)$ is a smooth manifold. Moreover, in this case the orbit space of $\mathcal{G}(A)$ is the unique source-simply connected Lie groupoid integrating $A$.

Proof. First, let $\operatorname{Mon}\left(P_{a} A\right)$ be the monodromy groupoid of the foliation introduced by homotopy of $A$-paths in $\S 2.1$. We will show that $\operatorname{Mon}\left(P_{a} A\right)$ is Morita equivalent to $\operatorname{Mon}\left(P_{0} A\right)$. Note that $P_{0} A$ is a submanifold of $P_{a} A$, so there is another groupoid $\left.\operatorname{Mon}\left(P_{a} A\right)\right|_{P_{o} A}$ over $P_{0} A$. We claim it is the same as $\operatorname{Mon}\left(P_{0} A\right)$. Namely, an A-homotopy $a(\epsilon, t)$ between two $A_{0}$ paths $a_{0}$ and $a_{1}$ can be homotopic to an $A_{0}$-homotopy $\tilde{a}(\epsilon, t)$ between $a_{0}$ and $a_{1}$. The idea is to divide $\tilde{a}$ into three parts.

(i) First deform $a_{0}$ to $a_{0}^{\tau}$ through $a_{0}(\epsilon, t)$ which is defined as $\left(1-\epsilon+\epsilon \tau^{\prime}(t)\right) a_{0}((1-\epsilon) t+\epsilon \tau(t))$, where $\tau$ is the reparametrization induced in $\S 2.1$.

(ii) Then deform $a_{0}^{\tau}$ to $a_{1}^{\tau}$ through $a(\epsilon, t)^{\tau}$.

(iii) Lastly, connect $a_{1}^{\tau}$ to $a_{1}$ through $a_{1}(\epsilon, t)$, which is defined as $a_{1}\left((1-\epsilon) \tau^{\prime}(t)+\epsilon\right) a_{1}(\epsilon t+(1-\epsilon) \tau(t))$. Then connect those three pieces by a similar method in the construction of concatenation (although it might be only piecewise smooth at the joints). Obviously, $\tilde{a}$ is a homotopy through $A_{0}$-paths and it is homotopic to a rescaling (over $\epsilon$ ) of $a(\epsilon, t)$ through the concatenation of $a_{0}((1-\lambda) \epsilon, t),\left(\lambda+(1-\lambda) \tau^{\prime}(t)\right) a\left(\epsilon, \lambda+(1-\lambda) \tau^{\prime}(t)\right)$ and $a_{1}((1-\lambda) \epsilon+\lambda, t)$. Eventually, we can smooth out everything to make the homotopy and the homotopy of homotopy both smooth so that they are as desired.

Then, it is routine to check that $\left.\operatorname{Mon}\left(P_{a} A\right)\right|_{P_{0} A}$ is Morita equivalent to $\operatorname{Mon}\left(P_{a} A\right)$ through $\mathbf{t}_{a}^{-1}\left(P_{0} A\right)$, where $\mathbf{t}_{a}$ is the target $\operatorname{Mon}\left(P_{a} A\right)$.

So the orbit space of $\mathcal{G}(A)$ can be realized as $P_{a} A / \operatorname{Mon}\left(P_{a} A\right)$. According to the main result in [CF03], $P_{a} A / \operatorname{Mon}\left(P_{a} A\right)$ is a smooth manifold if and only if $A$ is integrable and, if so, $P_{a} A / \operatorname{Mon}\left(P_{a} A\right)$ is the unique source-simply connected Lie groupoid integrating $A$.

Proof of Theorem 1.3. First of all, by the same argument given in the proof above, one can see that $\operatorname{Hol}\left(P_{0} A\right)=\left.\operatorname{Hol}\left(P_{a} A\right)\right|_{P_{0} A}$. Hence, $\operatorname{Hol}\left(P_{0} A\right)$ is Morita equivalent to $\operatorname{Hol}\left(P_{a} A\right)$. 


\section{INTEGRATING LIE ALGEBROIDS VIA STACKS}

Moreover, if the orbit space of a holonomy groupoid is a manifold then it is Morita equivalent to the holonomy groupoid itself (see [MM03]).

Hence, a differentiable stack $\mathcal{X}=B G$ presented by a holonomy groupoid $G$ is representable if and only if the orbit space $G_{0} / G_{1}$ is a smooth manifold. One direction is obvious because $G_{0} / G_{1} \rightrightarrows$ $G_{0} / G_{1}$ is Morita equivalent to $G=\left(G_{1} \rightrightarrows G_{0}\right)$ if the orbit space is a manifold. The converse direction is not hard to establish by examining the Morita equivalence diagram of $G$ and $\mathcal{X} \rightrightarrows \mathcal{X}$. The Morita bibundle has to be $G_{0}$ as $\mathcal{X}$ is a manifold. Therefore, $G_{0}$ is a principal $G$ bundle over $\mathcal{X}$. This implies that $G_{0} / G_{1}$ is the manifold $\mathcal{X}$.

Note that, in general, the orbit spaces of monodromy groupoids and holonomy groupoids of a foliation are the same. By Theorem 4.9 and argument above, we conclude that $A$ is integrable if and only if $\mathcal{H}(A)$ is representable and in this case, $\mathcal{H}(A)$ is $P_{a} A / \operatorname{Hol}\left(P_{a} A\right)$, the unique source-simply connected Lie groupoid integrating $A$.

Combining the proofs of Theorems 4.9 and 1.3, Theorem 1.5 follows naturally.

So far we have constructed $\mathcal{G}(A)$ and $\mathcal{H}(A)$ for every Lie algebroid $A$ and verified that they are Weinstein groupoids. Basically, we have done half of Theorem 1.2. For the other half of the proof, we first introduce some properties of Weinstein groupoids. Before doing so, we give an example.

Example $2\left(B \mathbb{Z}_{2}\right) . B \mathbb{Z}_{2}$ is a Weinstein group (i.e. its base space is a point) integrating the trivial Lie algebra 0 . The étale differentiable stack $B \mathbb{Z}_{2}$ is presented by $\mathbb{Z}_{2} \rightrightarrows p t$ (here $p t$ represents a point). We establish all of the structure maps on this presentation.

The source and target maps are just projections from $B \mathbb{Z}_{2}$ to a point. The multiplication $m$ is defined by

$$
m:\left(\mathbb{Z}_{2} \rightrightarrows p t\right) \times\left(\mathbb{Z}_{2} \rightrightarrows p t\right) \rightarrow\left(\mathbb{Z}_{2} \rightrightarrows p t\right), \quad \text { by } m(a, b)=a \cdot b,
$$

where $a, b \in \mathbb{Z}_{2}$. As $\mathbb{Z}_{2}$ is commutative, the multiplication is a groupoid homomorphism (hence gives rise to a stack homomorphism). It is easy to see that $m \circ(m \times i d)=m \circ(i d \times m)$, i.e. we can choose the 2-morphism $\alpha$ inside the associativity diagram to be $i d$.

The identity section $e$ is defined by

$$
e:(p t \rightrightarrows p t) \rightarrow\left(\mathbb{Z}_{2} \rightrightarrows p t\right), \quad \text { by } e(p t)=1,
$$

where 1 is the identity element in the trivial group $p t$ and $\mathbb{Z}_{2}$. The inverse $i$ is defined by

$$
i:\left(\mathbb{Z}_{2} \rightrightarrows p t\right) \rightarrow\left(\mathbb{Z}_{2} \rightrightarrows p t\right), \quad \text { by } i(a)=a^{-1},
$$

where $a \in \mathbb{Z}_{2}$. It is a groupoid homomorphism because $\mathbb{Z}_{2}$ is commutative.

It is routine to check whether these maps satisfy the axioms of Weinstein groupoids. The local Lie groupoid associated with $B \mathbb{Z}_{2}$ is just a point. Therefore, the Lie algebra of $B \mathbb{Z}_{2}$ is 0 . Moreover, note that we have only used the commutativity of $\mathbb{Z}_{2}$. So for any discrete commutative group $G$, $B G$ is a Weinstein group with Lie algebra 0 .

Example $3\left(\mathbb{Z}_{2} * B \mathbb{Z}_{2}\right)$. This is an example where Proposition 4.5 does not hold. Consider the groupoid $\Gamma=\left(\mathbb{Z}_{2} \times \mathbb{Z}_{2} \rightrightarrows \mathbb{Z}_{2}\right)$. It is an action groupoid with trivial $\mathbb{Z}_{2}$-action on $\mathbb{Z}_{2}$. We claim that the presented étale differential stack $B \Gamma$ is a Weinstein group. We establish all of the structure maps on the presentation $\Gamma$.

The source and target maps are projections to a point. The multiplication $m: \Gamma \times \Gamma \rightarrow \Gamma$ is defined by

$$
m\left(\left(g_{1}, a_{1}\right),\left(g_{2}, a_{2}\right)\right)=\left(g_{1} g_{2}, a_{1} a_{2}\right) .
$$

It is a groupoid morphism because $\mathbb{Z}_{2}$ (the second copy) is commutative. We have $m \circ(m \times i d)=$ $m \circ(i d \times m)$. However, we can construct a non-trivial 2-morphism $\alpha: \Gamma_{0}\left(=\mathbb{Z}_{2}\right) \times \Gamma_{0} \times \Gamma_{0} \rightarrow \Gamma_{1}$ 
defined by

$$
\alpha\left(g_{1}, g_{2}, g_{3}\right)=\left(g_{1} \cdot g_{2} \cdot g_{3}, g_{1} \cdot g_{2} \cdot g_{3}\right) .
$$

As the $\mathbb{Z}_{2}$ action on $\mathbb{Z}_{2}$ is trivial, we have $m \circ(m \times i d)=m \circ(i d \times m) \cdot \alpha$.

The identity section $e$ is defined by

$$
e:(p t \rightrightarrows p t) \rightarrow \Gamma, \quad \text { by } e(p t)=(1,1),
$$

where 1 is the identity element in $\mathbb{Z}_{2}$. The inverse $i$ is defined by

$$
i: \Gamma \rightarrow \Gamma, \quad \text { by } i(g, a)=\left(g^{-1}, a^{-1}\right) .
$$

It is a groupoid morphism because $\mathbb{Z}_{2}$ (the second copy) is commutative.

It is not hard to check whether $B \Gamma$ with these structures maps is a Weinstein group. However, it does not satisfy the further obstruction of the associativity described in Proposition 4.5, we found failure. Let $F_{i}$ be the six different ways of composing four elements as defined in Proposition 4.5, then the 2-morphisms $\alpha_{i}$ (basically coming from $\alpha$ ) satisfy

$$
F_{i+1}=F_{i} \cdot \alpha_{i}, \quad i=1, \ldots, 6\left(F_{7}=F_{1}\right) .
$$

However, $\alpha_{i}(1,1,1,-1)=(-1,-1)$ for all $i$ except that $\alpha_{2}=i d$. Therefore, $\alpha_{6} \circ \alpha_{5} \circ \cdots \circ \alpha_{1}(1,1$, $1,-1)=(-1,-1)$, which is not $i d(1,1,1,-1)=(-1,1)$.

\section{Weinstein groupoids and local groupoids}

In this section, we examine the relation between abstract Weinstein groupoids and local groupoids. Let us first show a useful lemma.

Lemma 5.1. Given any étale atlas $G_{0}$ of $\mathcal{G}$, there exists an open covering $\left\{M_{l}\right\}$ of $M$ such that the immersion $\bar{e}: M \rightarrow \mathcal{G}$ can be lifted to embeddings $e_{l}: M_{l} \rightarrow G_{0}$. On the overlap $M_{l} \cap M_{j}$, there exist an isomorphism $\varphi_{l j}: e_{j}\left(M_{j} \cap M_{l}\right) \rightarrow e_{l}\left(M_{j} \cap M_{l}\right)$, such that $\varphi_{l j} \circ e_{j}=e_{l}$ and the $\varphi_{l j}$ satisfy cocycle conditions.

Proof. Let $\left(E_{e}, J_{M}, J_{G}\right)$ be the HS bibundle presenting the immersion $\bar{e}: M \rightarrow \mathcal{G}$. As a right $G$-principal bundle over $M, E_{e}$ is locally trivial, i.e. we can pick an open covering $\left\{M_{l}\right\}$ so that $J_{M}$ has a section $\tau_{l}: M_{l} \rightarrow E_{e}$ when restricted to $M_{l}$. As $\bar{e}_{l}:=\left.\bar{e}\right|_{M_{l}}$ is an immersion (the composition of immersions $M_{l} \rightarrow M$ and $\bar{e}$ is still an immersion), it is not hard to see that $p r_{2}: M_{l} \times \mathcal{G}_{\mathcal{G}} G_{0} \rightarrow G_{0}$ transformed by base change $G_{0} \rightarrow \mathcal{G}$ is an immersion. Note that $e_{l}=J_{G} \tau_{l}: M_{l} \rightarrow G_{0}$ fits inside a similar diagram as (2).

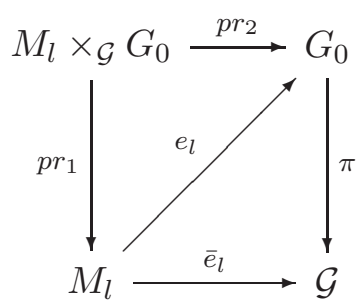

Following a similar argument as in the proof of Lemma 4.2, we can find a map $\alpha: M_{l} \times_{\mathcal{G}} G \rightarrow G_{1}$ such that

$$
e_{l} \circ p r_{1}=p r_{2} \cdot \alpha .
$$

As $\pi$ is étale, so is $p r_{1}$. Therefore $e_{l}$ is an immersion.

As an immersion is locally an embedding, we can choose an open covering $M_{i k}$ of $\left\{M_{l}\right\}$ so that $\left.e_{l}\right|_{M_{i k}}$ is actually an embedding. To simplify the notation, we can choose a finer covering $\left\{M_{l}\right\}$ at the beginning and make $e_{l}$ an embedding. Moreover, using the fact that $G$ acts on $E_{e}$ 


\section{INTEGRATING LIE ALGEBROIDS VIA STACKS}

transitively (fiberwise), it is not hard to find a local bisection $g_{l j}$ of $G_{1}:=G_{0} \times{ }_{\mathcal{G}} G_{0}$, such that $e_{l} \cdot g_{l j}=e_{j}$. Then $\varphi_{l j}=\cdot g_{l j}^{-1}$ satisfies that $\varphi_{l j} \circ e_{j}=e_{l}$. As $e_{l}$ are embeddings, $\phi_{l j}$ naturally satisfy the cocycle condition.

Before the proof of Theorem 1.4, we need a local statement.

Theorem 5.2. For every Weinstein groupoid $\mathcal{G}$, there exists an open covering $\left\{M_{l}\right\}$ of $M$ such that one can associate a local Lie groupoid $U_{l}$ over each open set $M_{l}$.

Proof. Let $\mathcal{G}$ be presented by $G=\left(G_{1} \rightrightarrows G_{0}\right)$, and $\left\{M_{l}\right\}$ be an open covering as in Lemma 5.1. Let $\left(E_{m}, J_{1}, J_{2}\right)$ be the HS bibundle from $G_{1} \times{ }_{M} G_{1} \rightrightarrows G_{0} \times_{M} G_{0}$ to $G$, which presents the stack morphism $\bar{m}: \mathcal{G} \times{ }_{M} \mathcal{G} \rightarrow \mathcal{G}$. Note that $M$ is the identity section, i.e.

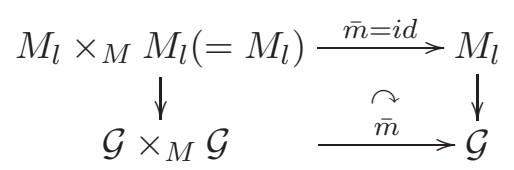

Translate this commutative diagram into groupoids. Then the composition of HS morphisms

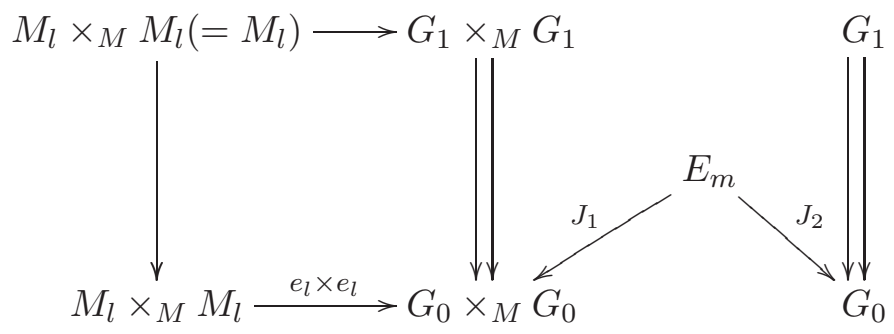

is the same (up to a 2-morphism) as $e_{l}: M_{l} \rightarrow G_{0}$. Therefore, composing the HS maps in (4) gives a HS bibundle $J_{1}^{-1}\left(\left(e_{l} \times e_{l}\right)\left(M_{l} \times{ }_{M} M_{l}\right)\right)$, which is isomorphic (as a HS bibundle) to $M_{l} \times_{G_{0}} G_{1}$, which represents the embedding $e_{l}$. Therefore, one can easily find a global section

$$
\sigma_{l}: M_{l} \rightarrow M_{l} \times_{G_{0}} G_{1} \cong J_{1}^{-1}\left(\left(e_{l} \times e_{l}\right)\left(M_{l} \times_{M} M_{l}\right)\right) \subset E_{m}
$$

defined by $x \mapsto\left(x, 1_{e_{l}(x)}\right)$. Furthermore, we have $J_{2} \circ \sigma_{l}\left(M_{l}\right)=e_{l}\left(M_{l}\right)$. As $G$ is an étale groupoid, $E_{m}$ is an étale principal bundle over $G_{0} \times_{M} G_{0}$. Hence, $J_{1}$ is a local diffeomorphism. Therefore, one can choose two open neighborhoods $V_{l} \subset U_{l}$ of $M_{l}$ in $G_{0}$ such that there exists a unique section $\sigma_{l}^{\prime}$ extending $\sigma_{l}$ over $\left(M_{l}=M_{l} \times_{M} M_{l} \subset\right) V_{l} \times_{M_{l}} V_{l}$ in $E_{m}$ and the image of $J_{2} \circ \sigma_{l}^{\prime}$ is $U_{l}$. The restriction of $\sigma_{l}^{\prime}$ on $M_{l}$ is exactly $\sigma_{l}$. As $U_{l} \rightrightarrows U_{l}$ acts freely and transitively fiberwise on $\sigma_{l}^{\prime}\left(V_{l} \times_{M_{l}} V_{l}\right)$ from the right, $\sigma_{l}^{\prime}\left(V_{l} \times_{M_{l}} V_{l}\right)$ can serve as a HS bibundle from $V_{l} \times_{M_{l}} V_{l}$ to $U_{l}$. (Here, we view manifolds as groupoids.) In fact, it is the same as the morphism

$$
m_{l}:=J_{2} \circ \sigma_{l}^{\prime}: V_{l} \times_{M_{l}} V_{l} \rightarrow U_{l} .
$$

By a similar method, we can define the inverse as follows. By (3), (4) and (5) in Definition 1.1, we have $\bar{i} \circ \bar{e}_{l}=\bar{e}_{l}$, so the following diagram commutes.

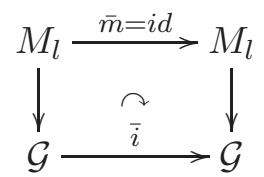

Suppose $\left(E_{i}, J_{1}, J_{2}\right)$ is the HS bibundle representing $\bar{i}$. Translate the above diagram into groupoids, 


\section{H.-H. Tseng And C. Zhu}

we have the composition of the following HS morphisms:

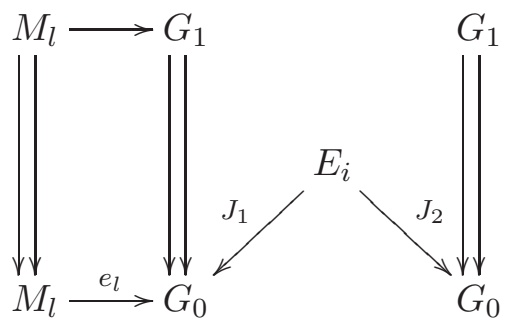

is the same (up to a 2-morphism) as $e_{l}: M_{l} \rightarrow G_{0}$. Therefore, composing the HS maps in (5) gives a HS bibundle $J_{1}^{-1}\left(e_{l}\left(M_{l}\right)\right)$ that is isomorphic (as a HS bibundle) to $M_{l} \times{ }_{G_{0}} G_{1}$, which represents the embedding $e_{l}$. Therefore, one can easily find a global section

$$
\tau_{l}: M_{l} \rightarrow M_{l} \times{ }_{G_{0}} G_{1} \cong J_{1}^{-1}\left(e_{l}\left(M_{l}\right)\right) \subset E_{i}
$$

defined by $x \mapsto\left(x, 1_{e_{l}(x)}\right)$. Furthermore, we have $J_{2} \circ \sigma_{l}\left(M_{l}\right)=e_{l}\left(M_{l}\right)$. As $G$ is an étale groupoid, $E_{i}$ is an étale principal bundle over $G_{0}$. Hence, $J_{1}$ is a local diffeomorphism. Therefore, one can choose an open neighborhood of $M_{l}$ in $G_{0}$, which we might assume as $U_{l}$ as well, such that there exists a unique section $\tau_{l}^{\prime}$ extending $\tau_{l}$ over $\left(M_{l} \subset\right) U_{l}$ in $E_{i}$ and the image of $J_{2} \circ \tau_{l}^{\prime}$ is in $U_{l}$. The restriction of $\tau_{l}^{\prime}$ on $M_{l}$ is exactly $\tau_{l}$. So we can define

$$
i_{l}:=J_{2} \circ \tau_{l}^{\prime}: U_{l} \rightarrow U_{l} .
$$

As $M$ is a manifold, examining the groupoid picture of maps $\overline{\mathbf{s}}$ and $\overline{\mathbf{t}}$, one finds that they actually come from two maps $\mathbf{s}$ and $\mathbf{t}$ from $G_{0}$ to $M$. Hence, we define source and target maps of $U_{l}$ as the restriction of $\mathbf{s}$ and $\mathbf{t}$ on $U_{l}$ and denote them by $\mathbf{s}_{l}$ and $\mathbf{t}_{l}$, respectively.

The 2-associative diagram of $\bar{m}$ tells us that $m_{l} \circ\left(m_{l} \times i d\right)$ and $m_{l} \circ\left(i d \times m_{l}\right)$ differ in the following way: there exists a smooth map from an open subset of $V_{l} \times_{M_{l}} V_{l} \times_{M_{l}} V_{l}$, over which both of the above maps are defined, to $G_{1}$ such that

$$
m_{l} \circ\left(m_{l} \times i d\right)=m_{l} \circ\left(i d \times m_{l}\right) \cdot \alpha .
$$

As the 2-morphism in the associative diagram restricting to $M$ is $i d$, we have

$$
\alpha(x, x, x)=1_{e_{l}(x)} .
$$

As $G$ is étale and $\alpha$ is smooth, the image of $\alpha$ is inside the identity section of $G_{1}$. Therefore, $m_{l}$ is associative.

It is not hard to verify other groupoid properties in a similar way by translating corresponding properties on $\mathcal{G}$ to $U_{l}$. Therefore, $U_{l}$ with maps defined above is a local Lie groupoid over $M_{l}$.

To prove the global result, we need the following proposition.

Proposition 5.3. Given $U_{l}$ and $U_{j}$ constructed as above (one can shrink them if necessary), there exists an isomorphism of local Lie groupoids $\tilde{\varphi}_{l j}: U_{j} \rightarrow U_{l}$ extending the isomorphism $\varphi_{l j}$ in Lemma 5.1. Moreover, $\tilde{\varphi}_{l j}$ also satisfy cocycle conditions.

Proof. As we restrict the discussion on $M_{l} \cap M_{j}$, we may assume that $M_{l}=M_{j}$. According to Lemma 5.1, there is a local bisection $g_{l j}$ of $G_{1}$ such that $e_{l} \cdot g_{l j}=e_{j}$. Extend the bisection $g_{l j}$ to $U_{l}$ (we denote the extension still by $g_{l j}$, and shrink $V_{k}$ and $U_{k}$ if necessary for $k=l, j$ ) so that

$$
\left(V_{l} \times_{M_{l}} V_{l}\right) \cdot\left(g_{l j} \times g_{l j}\right)=V_{j} \times_{M_{j}} V_{j} \quad \text { and } \quad U_{l} \cdot g_{l j}=U_{j} .
$$

Note that as $G_{1}$ is étale, the source map is an local isomorphism. Therefore, by choosing small enough neighborhoods of $M_{l}$, the extension of $g_{l j}$ is unique. Let $\tilde{\varphi}_{l j}=\cdot g_{l j}^{-1}$. Then it is naturally an extension of $\varphi_{l j}$. Moreover, by uniqueness of the extension, $\tilde{\varphi}_{l j}$ satisfy cocycle conditions as $\varphi_{l j}$ do. 


\section{INTEGRATING LIE ALGEBROIDS VIA STACKS}

Now we show that $\tilde{\varphi}_{l j}=\cdot g_{l j}$ is a morphism of local groupoids. It is not hard to see that $\cdot g_{l j}$ preserves source, target and identity embeddings. So we only have to show that

$$
i_{l} \cdot g_{l j}=i_{j}, \quad m_{l} \cdot g_{l j}=m_{j}
$$

For this purpose, we have to recall the construction of these two maps. $i_{l}$ is defined as $J_{2} \circ \tau_{l}^{\prime}$. As there is a global section of $J_{1}$ over $U_{l}$ in $E_{i}$, we have $J_{1}^{-1}\left(U_{l}\right) \cong U_{l} \times_{i_{l}, G_{0}} G_{1}$ as $G$ torsors. Under this isomorphism, we can write $\tau_{l}^{\prime}$ as

$$
\tau_{l}^{\prime}(x)=\left(x, 1_{e_{l}(x)}\right) .
$$

The $G$ action on $U_{l} \times_{i_{l}, G_{0}} G_{1}$ gives $\left(x, 1_{e_{l}(x)}\right) \cdot g_{l j}=\left(x, g_{l j}\right)$. Moreover, we have

$$
J_{2}\left(\left(x, g_{l j}\right)\right)=J_{2}\left(x, 1_{e_{j}(x)}\right)=\mathbf{s}_{G}\left(g_{l j}\right),
$$

where $\mathbf{s}_{G}$ is the source map of $G$. Combining all these, we have shown that $i_{l} \cdot g_{l j}=i_{j}$. The other identity for multiplications follows in a similar way.

Proof of Theorem 1.4. Now it is easy to construct $G_{\mathrm{loc}}$ as in the statement of the theorem. Note that the set of $\left\{U_{l}\right\}$ with isomorphisms $\varphi_{l j}$ which satisfy cocycle conditions serve as a chart system. Therefore, after gluing them together, we arrive at a global object $G_{\text {loc }}$. As $\varphi_{l j}$ are isomorphisms of local Lie groupoids, the local groupoid structures also glue together. Therefore, $G_{\text {loc }}$ is a local Lie groupoid.

If we choose two different open covering $\left\{M_{l}\right\}$ and $\left\{M_{l}^{\prime}\right\}$ of $M$ for the same étale atlas $G_{0}$ of $\mathcal{G}$, we arrive at two systems of local groupoids $\left\{U_{l}\right\}$ and $\left\{U_{l}^{\prime}\right\}$. As $\left\{M_{l}\right\}$ and $\left\{M_{l}^{\prime}\right\}$ are compatible chart systems for $M$, combining them and using Proposition 5.3, $\left\{U_{l}\right\}$ and $\left\{U_{l}^{\prime}\right\}$ are also compatible chart systems. Therefore, they glue into the same global object up to isomorphisms near the identity section.

If we choose two different étale atlases $G_{0}^{\prime}$ and $G_{0}^{\prime \prime}$ of $\mathcal{G}$, we can take their refinement $G_{0}=$ $G_{0}^{\prime} \times{ }_{\mathcal{G}} G_{0}^{\prime \prime}$ and we can take a fine enough open covering $\left\{M_{l}\right\}$ so that it embeds into all three atlases. As $G_{0} \rightarrow G_{0}^{\prime}$ is an étale covering, we can choose $U_{l}$ in $G_{0}^{\prime}$ small enough so that they still embed into $G_{0}$. So the groupoid constructed from the presentation $G_{0}$ with the covering $U_{l}$ is the same as the groupoid constructed from the presentation $G_{0}^{\prime}$ with the covering $U_{l}$. The same is true for $G_{0}^{\prime \prime}$ and $G_{0}$. Therefore, our local groupoid $G_{\text {loc }}$ is canonical.

We finish the proof of the Lie algebroid part in the next section.

\section{Weinstein groupoids and Lie algebroids}

In this section, we define the Lie algebroid of a Weinstein groupoid $\mathcal{G}$. An obvious choice is to define the Lie algebroid of $\mathcal{G}$ as the Lie algebroid of the local Lie groupoid $G_{\text {loc }}$.We give an equivalent definition in a more direct way.

Definition 6.1. Given a Weinstein groupoid $\mathcal{G}$ over $M$, there is a canonically associated Lie algebroid $A$ over $M$.

Proof. We just have to examine the second part of proof of Theorem 1.4 more carefully. Choose an étale groupoid presentation $G$ of $\mathcal{G}$ and an open covering $M_{l}$ as in Lemma 5.1. According to Theorem 1.4, we have a local groupoid $U_{l}$ and its Lie algebroid $A_{l}$ over each $M_{l}$. Differentiating the $\tilde{\varphi}_{l j}$ in Proposition 5.3, we can achieve algebroid isomorphisms $T \tilde{\varphi}_{l j}$, which also satisfy cocycle conditions. Therefore, using these data, we can glue the $A_{l}$ into a vector bundle $A$. Moreover, as the $T \tilde{\varphi}_{l j}$ are Lie algebroid isomorphisms, we can also glue the Lie algebroid structures. Therefore, $A$ is a Lie algebroid.

Following the same arguments as in the proof of Theorem 1.4, we can show uniqueness. If we choose a different presentation $G^{\prime}$ and a different open covering $M_{l}$, we can choose the refinement 


\section{H.-H. Tseng AND C. Zhu}

of these two systems and will arrive at a Lie algebroid which is glued from a refinement of both systems. Therefore, it is isomorphic to both Lie algebroids constructed from these two systems. Hence, the construction is canonical.

Then it is easy to see that the following proposition holds.

Proposition 6.2. Given a Weinstein groupoid $\mathcal{G}$, it has the same Lie algebroid as its associated local Lie groupoid $G_{\text {loc }}$.

Together with the Weinstein groupoid $\mathcal{G}(A)$ that we have constructed in $\S 4$, we are now ready to complete the proof of Theorem 1.2.

Proof of the second half of Theorem 1.2. We take the étale presentation $P$ of $\mathcal{G}(A)$ and $\mathcal{H}(A)$ as we constructed in $\S 2.1$. Let us recall how we construct local groupoids from $\mathcal{G}(A)$ and $\mathcal{H}(A)$.

In our case, the HS morphism corresponding to $\bar{m}$ is

$$
\left(E:=\mathbf{t}_{M}^{-1}\left(m\left(P \times_{M} P\right)\right) \cap \mathbf{s}_{M}^{-1}(P), m^{-1} \circ \mathbf{t}_{M}, \mathbf{s}_{M}\right) .
$$

The section $\sigma: M \rightarrow E$ is given by $x \mapsto 1_{0_{x}}$. Therefore, if we choose two small enough open neighborhoods $V \subset U$ of $M$ in $P$, the bibundle representing the multiplication $m_{V}$ is a section $\sigma^{\prime}$ over $V \times_{M} V$ of the map $m^{-1} \circ \mathbf{t}_{M}$ in $E$.

As the foliation $\mathcal{F}$ intersects each transversal slice only once, we can choose an open neighborhood $O$ of $M$ inside $P_{0} A$ so that the leaves of the restricted foliation $\left.\mathcal{F}\right|_{O}$ intersect $U$ only once. We denote the homotopy induced by $\left.\mathcal{F}\right|_{O}$ as $\sim_{O}$ and the holonomy induced by $\mathcal{F}_{O}$ by $\sim_{O}^{\text {hol }}$. Then there is a unique element $a \in U$ such that $a \sim_{O} a_{1} \odot a_{2}$. There exists a unique arrow $g: a_{1} \odot a_{2} \curvearrowleft a$ in $\operatorname{Mon}\left(P_{0} A\right)$ near the identity arrows at $1_{0_{x}}$ as the leaf of $\left.\mathcal{F}\right|_{O}$ is locally contractible.

Then we can choose the section $\sigma^{\prime}$ near $\sigma$ to be

$$
\sigma^{\prime}:\left(a_{1}, a_{2}\right) \mapsto g .
$$

So the multiplication $m_{V}$ on $U$ is

$$
m_{V}\left(a_{1}, a_{2}\right)=a\left(\sim_{O} a_{1} \odot a_{2}\right) .
$$

As the leaves of $\mathcal{F}$ intersect $U$ only once, $a$ has to be the unique element in $U$ such that $a \sim \sim_{O}^{\text {hol }} a_{1} \odot a_{2}$. It is not hard to verify that both Weinstein groupoids give the same local Lie groupoid structure on $U$.

Moreover, $U=O / \sim_{O}$ is exactly the local groupoid constructed in [CF03, $\left.\S 5\right]$, which has Lie algebroid $A$. Therefore, $\mathcal{G}(A)$ and $\mathcal{H}(A)$ have the same local Lie groupoid and their Lie algebroids are both $A$.

\section{ACKNOWLEDGEMENTS}

We thank K. Behrend, H. Bursztyn, M. Crainic, T. Graber, D. Metzler, I. Moerdijk, J. Mrčun, A. Weinstein, P. Xu and Marco Zambon for very helpful discussions and suggestions. We also appreciate various suggestions from the referee and the editors.

\section{REFERENCES}

AM84 R. Almeida and P. Molino, Suites d'Atiyah, feuilletages et quantification géomètrique, in Séminaire de géomètrie différentielle (Université des Sciences et Techniques de Languedoc, Montpellier, 1984), 39-59.

AM85 R. Almeida and P. Molino, Suites d'Atiyah et feuilletages transversalement complets, C. R. Acad. Sci. Paris Sér. I Math. 300 (1985), 13-15. 


\section{INTEGRATING LIE ALGEBROIDS VIA STACKS}

AGV72 M. Artin, A. Grothendieck and J. L. Verdier, Théorie des topos et cohomologie étale des schémas. Tome 1: Théorie des topos, in Séminaire de Géométrie Algébrique du Bois-Marie 1963-1964 (SGA 4), eds M. Artin et al., Lecture Notes in Mathematics, vol. 269 (Springer, Berlin, 1972).

BEFFGK K. Behrend, D. Edidin, B. Fantechi, W. Fulton, L. Göttsche and A. Kresch, Introduction to stacks, in preparation.

BX K. Behrend and P. Xu, Differentiable stacks and gerbes, in preparation.

BX03 K. Behrend and P. Xu, $S^{1}$-bundles and gerbes over differentiable stacks, C. R. Math. Acad. Sci. Paris 336 (2003), 163-168.

BW04 H. Bursztyn and A. Weinstein, Picard groups in Poisson geometry, Mosc. Math. J. 4 (2004), 39-66.

CW99 A. Cannas da Silva and A. Weinstein, Geometric models for noncommutative algebras, Berkeley Mathematics Lecture Notes, vol. 10 (American Mathematical Society, Providence, RI, 1999).

CF01 A. S. Cattaneo and G. Felder, Poisson sigma models and symplectic groupoids, in Quantization of singular symplectic quotients, Progress in Mathematics, vol. 198 (Birkhäuser, Basel, 2001), 61-93.

CDW87 A. Coste, P. Dazord and A. Weinstein, Groupoïdes symplectiques, in Publications du Département de Mathématiques. Nouvelle Série. A (2), vol. 87 (Université Claude-Bernard, Lyon, 1987), i-ii, $1-62$.

CF03 M. Crainic and R. L. Fernandes, Integrability of Lie brackets, Ann. of Math. (2) 157 (2003), $575-620$.

CF04 M. Crainic and R. L. Fernandes, Integrability of Poisson brackets, J. Differential Geom. 66 (2004), $71-137$.

CZ04 M. Crainic and C. Zhu, Integrability of Jacobi structures, Preprint (2004), arXiv:math.DG/0403268.

Daz90 P. Dazord, Groupoïdes symplectiques et troisième théorème de Lie "non linéaire", in Géométrie symplectique et mécanique (La Grande Motte, 1988), Lecture Notes in Mathematics, vol. 1416 (Springer, Berlin, 1990), 39-74.

Deb00 C. Debord, Groupö̈des d'holonomie de feuilletages singuliers, C. R. Acad. Sci. Paris Sér. I Math. 330 (2000), 361-364.

DM69 P. Deligne and D. Mumford, The irreducibility of the space of curves of given genus, Publ. Math. Inst. Hautes Études Sci. 36 (1969), 75-109.

Hae84 A. Haefliger, Groupoïdes d'holonomie et classifiants, in Transversal structure of foliations (Toulouse, 1982), Astérisque 116 (1984), 70-97.

Kar86 M. V. Karasëv, Poisson algebras of symmetries and asymptotic behavior of spectral series, Funktsional. Anal. i Prilozhen. 20 (1986), 21-32.

LM00 G. Laumon and L. Moret-Bailly, Champs algébriques (Springer, Berlin, 2000).

Mac87 K. Mackenzie, Lie groupoids and Lie algebroids in differential geometry, London Mathematical Society Lecture Note Series, vol. 124 (Cambridge University Press, Cambridge, 1987).

Met03 D. Metzler, Topological and smooth stacks, Preprint (2003), math.DG/0306176.

MM03 I. Moerdijk and J. Mrčun, Introduction to foliations and Lie groupoids, Cambridge Studies in Advanced Mathematics, vol. 91 (Cambridge University Press, Cambridge, 2003).

MRW87 P. S. Muhly, J. N. Renault and D. P. Williams, Equivalence and isomorphism for groupoid $C^{*}$-algebras, J. Operator Theory 17 (1987), 3-22.

Nis00 V. Nistor, Groupoids and the integration of Lie algebroids, J. Math. Soc. Japan 52 (2000), 847-868.

Pra68 J. Pradines, Troisième théorème de Lie les groupoïdes différentiables, C. R. Acad. Sci. Paris Sér. A-B 267 (1968), A21-A23.

Pro96 D. A. Pronk, Etendues and stacks as bicategories of fractions, Compositio Math. 102 (1996), 243-303.

van84 W. T. van Est, Rapport sur les $S$-atlas, in Transversal structure of foliations (Toulouse, 1982), Astérisque 116 (1984), 235-292. 


\section{INTEGRATING LIE ALGEBROIDS VIA STACKS}

Vis89 A. Vistoli, Intersection theory on algebraic stacks and on their moduli spaces, Invent. Math. 97 (1989), 613-670.

Vis02 A. Vistoli, Topological stacks (a talk given at MSRI in 2002), available at: http://www.msri.org/publications/ln/msri/2002/introstacks/vistoli/1/index.html.

Wei83 A. Weinstein, The local structure of Poisson manifolds, J. Differential Geom. 18 (1983), 523-557.

Wei87 A. Weinstein, Symplectic groupoids and Poisson manifolds, Bull. Amer. Math. Soc. (N.S.) 16 (1987), 101-104.

Wei89 A. Weinstein, Blowing up realizations of Heisenberg-Poisson manifolds, Bull. Sci. Math. 113 (1989), 381-406.

Wei03 A. Weinstein, Integrating the nonintegrable, lecture given at conference 'FeuilletagesQuantification géométrique', 2003.

Xu91a P. Xu, Morita equivalence of Poisson manifolds, Comm. Math. Phys. 142 (1991), 493-509.

Xu91b P. Xu, Morita equivalent symplectic groupoids, in Symplectic geometry, groupoids, and integrable systems (Berkeley, CA, 1989), Mathematical Science Research Institute Publications, vol. 20 (Springer, New York, 1991), 291-311.

Zhu04 C. Zhu, Integrating Lie algebroids via stacks and applications to Jacobi manifolds, PhD thesis, U.C. Berkeley (2004), math.DG/0505158.

Hsian-Hua Tseng hhtseng@math.berkeley.edu

Department of Mathematics, University of California, Berkeley, CA 94720, USA

Chenchang Zhu zhu@math.ethz.ch

Department Mathematik, ETH, Rämistrasse 101, 8092 Zürich, Switzerland 